\title{
MWIR HGCDTE PHOTODIODES BASED ON HIGH-DENSITY PLASMA- INDUCED TYPE CONVERSION
}

\author{
B.A. Park, C.A. Musca, R.J. Westerhout, J. Antoszewski, J.M. Dell, L. Faraone \\ School of Electrical, Electronic and Computer Engineering, The University of Western Australia, Crawley \\ Western Australia 6009, Australia
}

PACS: $85.60,71.55 . \mathrm{Gs}$

\begin{abstract}
A novel fabrication process for planar n-on-p junction $\mathrm{HgCdTe}$ infrared photodiodes has previously been demonstrated based on $\mathrm{H}_{2} / \mathrm{CH}_{4}$ plasma-induced type conversion in a parallel-plate reactive ion etching (RIE) tool. In this work, it is demonstrated that high-performance photodiodes can also be fabricated based on $\mathrm{H}_{2} / \mathrm{CH}_{4} / \mathrm{Ar}$ plasma-induced type conversion in an inductively coupled plasma (ICP) RIE plasma tool. This tool has significantly greater control over the plasma condition, and the plasma condition for the type conversion process is significantly different to that associated with the junction formation process that has previously been demonstrated based on the parallel-plate RIE reactor. Photodiodes have been fabricated based on different ICPRIE plasma process conditions, and characterised by current-voltage and noise measurements. The performance characteristics of these photodiodes are comparable to those reported for diodes based on established fabrication processes based on ion implantation or ion beam milling. The effects of the ICPRIE process parameters used for junction formation on the respective photodiode performance characteristics are investigated in terms of the plasma-induced type conversion mechanisms.
\end{abstract}




\section{INTRODUCTION}

$\mathrm{HgCdTe}$ is the preferred material for fabrication of high-performance infrared (IR) detectors in the midwave (MW) $(3-5 \mu \mathrm{m})$ and long-wave (LW) $(8-14 \mu \mathrm{m})$ IR bands due to a number of superior fundamental properties, despite difficulties in material growth and device fabrication processes primarily associated with the low damage threshold [1]. Traditional methods for fabricating n-on-p HgCdTe junction photodiode arrays are typically based on junction formation by ion implantation [2] or ion beam milling (IBM) [3]. With developments in epitaxial growth technology, more complicated and advanced fabrication processes are now commonly based on grown junctions and high-density reactive ion etching (RIE) processes to delineate and electrically isolate array elements [4].

It has previously been shown that exposure of p-type $\mathrm{HgCdTe}$ to energetic particles, such as those involved in RIE or IBM, induces modification to the electrical properties of the material, and more specifically causes p-to-n type conversion [5]. This type conversion phenomenon is generally an undesirable side-effect of plasma processing for etching applications, but can also be exploited for controlled junction formation. The type conversion mechanism is generally believed to be associated with diffusion of interstitial $\mathrm{Hg}$ which is liberated by the ion bombardment [5] [6], in addition to incorporation of hydrogen from the plasma [7].

This plasma-induced type conversion phenomenon has previously been demonstrated as a novel basis for planar n-on-p junction formation as part of a photodiode fabrication process [8]. Compared to fabrication processes for grown junctions, this simpler planar process does not necessitate dry etching processes to delineate device elements. This process has also advantages over the traditional junction formation techniques based on ion implantation or IBM. It does not require a post-process anneal to activate dopants and ameliorate ion-induced damage, and allows the passivation layer to be used as a mask for the junction 
formation process so that the surface need not be exposed to atmosphere. These previously demonstrated photodiodes were based on type conversion by exposure to $\mathrm{H}_{2} / \mathrm{CH}_{4}$ plasma in a parallel-plate RIE tool.

More advanced high-density RIE tools which incorporate a secondary power source, such as inductively coupled plasma (ICP), are now available. These hybrid tools are capable of generating plasma at lower pressures and of higher density than is possible using the basic parallel-plate system, in addition to exercising greater control over the plasma condition [9]. Given the extremely large parameter space and relatively limited understanding of plasma-induced type conversion mechanisms, there is much more work to be done before good control of this ICPRIE-induced type conversion process can be obtained.

In the previous work that originally developed the plasma-induced junction formation process based on the parallel-plate RIE tool, an investigation of how changing the plasma process parameters will affect the type conversion process or the photodiode performance had not yet been performed. More recently, using the hybrid ICPRIE tool, a parametric investigation of the ICPRIE-induced type conversion process was performed based on carrier transport characterisation of the p-to-n type converted layer. This was achieved using van der Pauw samples exposed to a variety of ICPRIE conditions, which were characterised by quantitative mobility spectrum analysis of variable-magnetic-field Hall and resistivity measurements. This previous work identified that the ICPRIE-induced type conversion process was most sensitive to the temperature during processing. Increased levels of RIE and/or ICP power were also found to be positively related to the type conversion depth [10]. These observations are consistent with the previously proposed hypothesis of a diffusion-based type conversion mechanism. However, it was found to be surprisingly difficult to achieve an ICPRIE-induced junction depth greater than $1 \mu \mathrm{m}$ in the vacancy-doped $\mathrm{Hg}_{0.7} \mathrm{Cd}_{0.3} \mathrm{Te}$ material.

For the first time, n-on-p $\mathrm{Hg}_{0.7} \mathrm{Cd}_{0.3}$ Te photodiodes have been fabricated in an ICPRIE reactor using $\mathrm{H}_{2} / \mathrm{CH}_{4} / \mathrm{Ar}$ ICPRIE-induced junction formation. Compared to the plasma conditions used in the previously 
reported process based on the parallel-plate RIE tool, this new process involved significantly higher plasma density, lower process pressure, addition of Ar to the plasma gas mixture, and higher sample temperature during junction formation. The effect of varying plasma conditions for the junction formation process on the performance of the associated photodiodes had previously not been reported. In this work, photodiode characterization results are presented for a number of junctions formed under different ICPRIE conditions. The current-voltage curves and noise characteristics of the photodiodes are analysed to investigate the probable differences in the fundamental properties of the junctions, and how these are controlled by the ICPRIE-induced type conversion process.

\section{DEVICE FABRICATION}

Starting material was vacancy-doped $\mathrm{Hg}_{0.7} \mathrm{Cd}_{0.3} \mathrm{Te}$ grown by liquid phase epitaxy, purchased from Fermionics Corporation (CA, USA). The manufacturer specified vacancy concentration $\mathrm{p}=1 \times 10^{16} \mathrm{~cm}^{-3}$ and nominal cutoff wavelength of $5.2 \mu \mathrm{m}$ at $77 \mathrm{~K}$. This process flow was previously developed based on the RIE reactor and has previously been described [8] [11]. The epilayer was thinned to about $10 \mu \mathrm{m}$ in $\mathrm{Br}_{2} /$ methanol solution, then molecular beam epitaxy was used to deposit a $100 \mathrm{~nm}$ thick passivation layer of CdTe. Photolithography and wet etching using $\mathrm{Br}_{2} / \mathrm{HBr}$ was used to define windows in this passivation layer for the junction formation step. The photoresist was removed prior to ICPRIE-induced type conversion. Plasma process conditions for the junction formation step associated with each photodiode array are summarized in Table I, including the plasma conditions previously developed for the RIE tool. It should be noted that the CdTe passivation layer was exposed to and etched by the $\mathrm{H}_{2} / \mathrm{CH}_{4} / \mathrm{Ar}$ plasma during the junction formation process.

Table I - Plasma-induced junction formation conditions for each sample. The conditions used for the RIEbased process are included for reference.

\begin{tabular}{|l|l|l|l|l|l|l|}
\hline $\begin{array}{l}\text { Diode } \\
\text { set }\end{array}$ & $\begin{array}{l}\text { Temperature } \\
(\mathbf{C})\end{array}$ & $\begin{array}{l}\mathbf{H}_{2} / \mathbf{C H}_{4} / \mathbf{A r} \\
\text { flow }(\mathbf{s c c m})\end{array}$ & $\begin{array}{l}\text { Process pressure } \\
\text { (mTorr) }\end{array}$ & $\begin{array}{l}\text { RIE } \\
\text { power (W) }\end{array}$ & $\begin{array}{l}\text { ICP } \\
\text { power (W) }\end{array}$ & $\begin{array}{l}\text { Process } \\
\text { time (s) }\end{array}$ \\
\hline A & 20 & $60 / 10 / 106$ & 80 & 100 & 250 & 30 \\
\hline B & 70 & $60 / 5 / 106$ & 15 & 50 & 250 & 30 \\
\hline C & 70 & $60 / 5 / 106$ & 15 & 40 & 175 & 45 \\
\hline Ref:RIE & 24 & $54 / 18 / 0$ & 100 & 100 & 0 & 120 \\
\hline
\end{tabular}


After the ICPRIE-induced junction formation process, an insulation layer was deposited over the CdTe passivation layer. For photodiode arrays A and B, this insulation layer was 200nm of thermally-deposited $\mathrm{ZnS}$, with the sample held at $50^{\circ} \mathrm{C}$. For photodiode array $\mathrm{C}$, this insulation layer was a layer of $\mathrm{SiN}_{\mathrm{x}}$ deposited by plasma enhanced chemical vapour deposition (PECVD), and involved holding the sample temperature at $125^{\circ} \mathrm{C}$ for about 15 minutes. It is possible that this temperature cycling may have affected the junction properties and assisted in-diffusion of the CdTe passivation layer, which may affect the photodiode performance. In previous work, a post-process bake for about one week at $80^{\circ} \mathrm{C}$ was reported to slightly improve the performance of photodiodes based on RIE-induced junction formation and using a $\mathrm{CdTe} / \mathrm{ZnS}$ passivation/insulation layer. This was attributed to in-diffusion of the CdTe passivation layer [12]. The difference in the insulation layers and deposition processes, particularly the potential effects of temperature cycling and the role of hydrogen in the PECVD process for $\mathrm{SiN}_{\mathrm{x}}$ deposition, should be considered when comparing and evaluating the performance of the different photodiode arrays. The final device structures are illustrated in Fig. 1 [13].

Devices were manually bonded out to a chip carrier using silver epoxy and gold wire. This bonding process is difficult, and the yield of successfully bonded photodiodes and gates was correspondingly low. The photodiode arrays were then mounted in a cryostat under a cold shield and placed under vacuum for characterization.

\section{CHARACTERISATION AND ANALYSIS}

An HP4156A semiconductor parameter analyzer was used to perform current-voltage (I-V) measurements at temperatures from $77 \mathrm{~K}$ to $300 \mathrm{~K}$. These $\mathrm{I}-\mathrm{V}$ characteristics can be analysed to characterise the photodiode performance. This photodiode performance is degraded by dark currents, which act to reduce the dynamic resistance of the photodiode and contribute to noise. Each dark current mechanism, including diffusion, generation-recombination (g-r), trap-assisted tunneling (TAT), and band-to-band (BTB) tunneling, has a distinct dependence on both junction bias and temperature [14] [15] [16]. 
Noise measurements were performed in a Faraday cage using an in-house-built ultra-low noise transimpedence amplifier and HP35665A dynamic signal analyzer. This amplifier has a gain of $10^{10}$, bandwidth of $17.8 \mathrm{kHz}$, and input noise current of $1.7 \times 10^{-30} \mathrm{~A}^{2} / \mathrm{Hz} \quad(1.3 \mathrm{fA} / \sqrt{\mathrm{Hz}})$ [13]. The noise characteristics of the photodiode are strongly related to the performance, with previous work having positively correlated increased photodiode noise with dark current, and specifically the TAT current mechanism [15] [17].

The dynamic-resistance-area-product $\left(\mathrm{R}_{\mathrm{d}} \mathrm{A}\right)$ curves corresponding to the measured I-V characteristics (corrected for series resistance) for the best photodiodes from each array are shown in Fig. 2 to Fig. 5, and noise measurements at $77 \mathrm{~K}$ shown in Fig. 6 and Fig. 7. The $\mathrm{R}_{\mathrm{d}} \mathrm{A}$ products are calculated based on the photomask dimensions for the junction.

The dotted lines in Fig. 2 to Fig. 5 show the theoretical behaviour of each dark current mechanism, which is based on models developed in previously published works [15] [18] [19]. The quantitative accuracy of the modeled fit to the $\mathrm{R}_{\mathrm{d}} \mathrm{A}$ at any given temperature is limited by the large number of variables. Furthermore, many of these variables, specifically including the location of the trap level in the material bandgap and the minority carrier lifetime, are not accurately known. The theoretical temperature dependence of each dark current mechanism, shown for photodiodes from sample C and sample B in Fig. 4 and Fig. 5, respectively, is intended only to indicate which mechanisms are dominant over specific temperature ranges. A complete investigation of dark current modeling is beyond the scope of this paper, however these dark current models have been found to be very useful to obtain a qualitative indication of the relative influence of each dark current mechanism. In the following paragraphs, the $R_{d} A$ and noise properties measured from photodiodes from each sample array are discussed and compared. 
The highest measured zero-bias-dynamic-resistance-area-product $\left(\mathrm{R}_{0} \mathrm{~A}\right)$ at $77 \mathrm{~K}$ from the photodiodes fabricated in this work based on ICPRIE-induced type conversion was $2.5 \times 10^{6} \Omega . \mathrm{cm}^{2}$ from sample C, as shown in Fig. 2 and Fig. 4. This $\mathrm{R}_{0} \mathrm{~A}$ value and the noise characteristics shown in Fig. 6 are similar to the best figures that have been previously reported for photodiodes based on the original RIE-induced type conversion process [8] [20], and comparable to high-performance MWIR photodiodes based on established fabrication processes of ion implantation [21] or ion beam milling [22]. This is a very promising result for a process in relatively early stages of development.

Based on the measured results and modeled fits shown in Fig. 2 and Fig. 4, photodiode A01 from sample $\mathrm{C}$ is diffusion-limited in forward bias at $77 \mathrm{~K}$, which is consistent with behaviour expected from highperformance photodiodes. The temperature-dependence of $\mathrm{R}_{0} \mathrm{~A}$ is consistent with diffusion-limited performance down to approximately $150 \mathrm{~K}$, possibly with some contribution from the non-fundamental g-r mechanism. Relatively little trap-assisted-tunneling (TAT) current was observed for this photodiode in reverse bias, which is consistent with good surface passivation and relatively low levels of trap density at the junction surface or in the bulk.

Compared to the junction formation process for sample $\mathrm{C}$, the ICPRIE conditions used to induce the junctions associated with sample B involved higher levels of RIE and ICP power, which are primarily expected to increase the acceleration of ions from the plasma and increase the plasma density, respectively. It is reasonable to expect that increased ion bombardment energy should result in increased electrical damage to the exposed $\mathrm{HgCdTe}$; specifically including increased trap density. The tradeoff between decreasing the levels of power and increasing the process time used to induce junctions in sample $\mathrm{C}$ compared to sample B was found to result in a similar physical etch depth (approximately $0.1 \mu \mathrm{m}$ ) in each junction area. Comparing the measured performance of photodiodes from sample B to that obtained from sample $C$, the $R_{d} A$ of photodiodes from sample $B$ was approximately an order of magnitude lower. The noise from photodiodes from sample B was approximately an order of magnitude higher, as shown in Fig. 
7. The dark current modeling results shown in Fig. 3 indicate that this was due to significantly greater TAT dark current in photodiodes from sample B, which was most likely due to enhanced electric field and/or trap density at the surface. This is consistent with increasing RIE power being associated with increased damage to exposed $\mathrm{HgCdTe}$, while the trade-off between the power and time for the ICPRIEinduced type conversion process was expected to liberate a similar dose of interstitial $\mathrm{Hg}$ to diffuse into the resultant junction area. It is also consistent with the hypothesis that increased photodiode noise is associated with increased TAT current and lower performance.

The photodiodes from sample A were formed by exposure to ICPRIE at lower temperature, higher pressure, and higher levels of both RIE and ICP power compared to photodiodes from sample B. The measured results for photodiodes from sample A are not reproduced here, but were consistent with decreased $\mathrm{R}_{\mathrm{d}} \mathrm{A}$ and increased TAT current compared to photodiodes from the other samples. Based on previously published carrier transport characterisation work, the junction depth of the photodiodes from sample A was expected to be approximately $0.1-0.4 \mu \mathrm{m}$, which is less than the typical target depth of approximately $1 \mu \mathrm{m}[23]$.

The higher sample temperature and lower levels of RIE during ICPRIE used to form the better photodiodes from samples B and C were expected to be associated with increased junction depth and lower levels of damage, respectively. Higher pressure was expected to cause a significant increase in the physical etch rate, which resulted in the etched junction recess depth for sample A being approximately double that of samples B and C. As this recess depth increases, the integrity surface passivation is more likely to be compromised, which may result in increased electric field and thus tunneling currents at the junction perimeter. These expected relationships between the ICPRIE process parameters, the junction properties, and the photodiode performance, were all consistent with the measured photodiode performance results. 


\section{CONCLUSIONS}

For the first time, high-performance n-on-p $\mathrm{Hg}_{0.7} \mathrm{Cd}_{0.3}$ Te photodiodes have been fabricated based on a number of different ICPRIE conditions for plasma-induced junction formation. The ICPRIE conditions used for the junction formation step were significantly different from the plasma conditions used in the previously demonstrated process in the older parallel-plate RIE tool. Performance of these photodiodes is generally limited by the surface condition rather than the quality of the n-on-p junction. The best measured $\mathrm{R}_{0} \mathrm{~A}$ for these novel ICPRIE-induced n-on-p planar photodiodes measured in the dark at $77 \mathrm{~K}$ was $2.5 \times 10^{6}$ $\Omega . \mathrm{cm}^{2}$, which is comparable to reported results for high-performance photodiodes fabricated by established junction formation techniques such as ion implantation. Changes to the ICPRIE conditions that have been associated with improved photodiode performance include increasing the sample temperature, decreasing the process pressure, and reducing the levels of RIE and ICP power. These changes are expected to increase the plasma-induced junction depth, and/or decrease the degree of plasma-induced damage to the surface and bulk. The major challenge in these tradeoffs was to form n-on-p junctions of sufficient depth to achieve high-quality bulk n-type material, while minimising the level of plasmainduced damage. This goal was achieved, as shown by the consistent measured improvement to the photodiode performance with each refinement to the ICPRIE conditions for junction formation. This novel photodiode fabrication technology is simpler than established methods such as ion implantation or epitaxially-grown junctions, and shows great promise for further improvement in photodiode performance. 


\section{REFERENCES}

[1] Rogalski, A., Toward third generation $\mathrm{HgCdTe}$ infrared detectors. Journal of Alloys and Compounds. 371(1-2): p. 53-57. (2004)

[2] Arias, J.M., Pasko, J.G., Zandian, M., Shin, S.H., Williams, G.M., Bubulac, L.O., Dewames, R.E., and Tennant, W.E., Planar P-on-N Hgcdte Heterostructure Photovoltaic Detectors. Applied Physics Letters. 62(9): p. 976-977. (1993)

[3] Bahir, G., Garber, V., and Rosenfeld, D., Planar p-on-n HgCdTe heterostructure infrared photodiodes. Applied Physics Letters. 78(10): p. 1331-1333. (2001)

[4] Bornfreund, R., Rosbeck, J.P., Thai, Y.N., Smith, E.P., Lofgreen, D.D., Vilela, M.F., Buell, A.A., Newton, M.D., Kosai, K., Johnson, S.M., De Leon, T., Jensen, J.E., and Tidrow, M.Z., Highperformance LWIR MBE-grown $\mathrm{HgCdTe/Si} \mathrm{focal} \mathrm{plane} \mathrm{arrays.} \mathrm{Journal} \mathrm{of} \mathrm{Electronic} \mathrm{Materials.}$ 36(8): p. 1085-1091. (2007)

[5] Belas, E., Franc, J., Toth, A., Moravec, P., Grill, R., Sitter, H., and Hoschl, P., Type conversion of p- $(\mathrm{HgCd}) \mathrm{Te}$ using $\mathrm{H}_{2} / \mathrm{CH}_{4}$ and Ar reactive ion etching. Semiconductor Science and Technology. 11(7): p. 1116-1120. (1996)

[6] Eddy, C.R., Dobisz, E.A., Meyer, J.R., and Hoffman, C.A., Electron cyclotron resonance reactive ion etching of fine features in $\mathrm{HgCdTe}$ using $\mathrm{CH}_{4} / \mathrm{H}_{2}$ plasmas. Journal of Vacuum Science \& Technology A. 11: p. 1763-1767. (1993)

[7] White, J., Pal, R., Dell, J.M., Musca, C.A., Antoszewski, J., Faraone, L., and Burke, P., P-to-n type-conversion mechanisms for $\mathrm{HgCdTe}$ exposed to $\mathrm{H}_{2} / \mathrm{CH}_{4}$ plasmas. Journal of Electronic Materials. 30(6): p. 762-767. (2001)

[8] Dell, J.M., Antoszewski, J., Rais, M.H., Musca, C., White, J.K., Nener, B.D., and Faraone, L., $\mathrm{HgCdTe}$ mid-wavelength IR photovoltaic detectors fabricated using plasma induced junction technology. Journal of Electronic Materials. 29(6): p. 841-848. (2000)

[9] Stoltz, A.J., Sperry, M.J., Benson, J.D., Varesi, J.B., Martinka, M., Almeida, L.A., Boyd, P.R., and Dinan, J.H., A Langmuir probe investigation of electron cyclotron resonance argon-hydrogen plasmas. Journal of Electronic Materials. 34(6): p. 733-739. (2005)

[10] Park, B.A., Musca, C.A., Antoszewski, J., Dell, J.M., and Faraone, L., Effect of high-density plasma process parameters on carrier transport properties in a p-to-n type converted $\mathrm{Hg}_{0.7} \mathrm{Cd} d_{0.3}$ Te layer. Journal of Electronic Materials. 36(8): p. 913-918. (2007)

[11] Nguyen, T., Musca, C.A., Dell, J.M., Antoszewski, J., and Faraone, L., HgCdTe long-wavelength infrared photovoltaic detectors fabricated using plasma-induced junction formation technology. Journal of Electronic Materials. 32(7): p. 615. (2003)

[12] White, J.K., Antoszewski, J., Pal, R., Musca, C.A., Dell, J.M., Faraone, L., and Piotrowski, J., Passivation effects on reactive-ion-etch-formed n-on-p junctions in HgCdTe. Journal of Electronic Materials. 31(7): p. 743-748. (2002)

[13] Westerhout, R.J., Musca, C.A., Antoszewski, J., Dell, J.M., and Faraone, L., Investigation of 1/f noise mechanisms in midwave infrared $\mathrm{HgCdTe}$ gated photodiodes. Journal of Electronic Materials. 36(8): p. 884-889. (2007)

[14] Nemirovsky, Y., Rosenfeld, D., Adar, R., and Kornfeld, A., Tunneling and dark currents in $\mathrm{HgCdTe}$ photodiodes. Journal of Vacuum Science \& Technology a-Vacuum Surfaces and Films. 7(2): p. 528-535. (1989)

[15] Nemirovsky, Y. and Unikovsky, A., Tunneling and 1/f noise currents in HgCdTe photodiodes. Journal of Vacuum Science \& Technology B. 10(4): p. 1602-1610. (1992)

[16] Rosbeck, J.P., Starr, R.E., Price, S.L., and Riley, K.J., Background and temperature dependent current-voltage characteristics of HgCdTe photodiodes. Journal of Applied Physics. 53(9): $\mathrm{p}$. 6430-6440. (1982) 
[17] Bajaj, J., Blazejewski, E.R., Williams, G.M., and De Wames, R.E., Noise (1/f) and dark currents in midwavelength infrared PACE-1 HgCdTe photodiodes. Journal of Vacuum Science \& Technology B. 10: p. 192-194. (1992)

[18] Nguyen, T., Musca, C.A., Dell, J.M., Antoszewski, J., and Faraone, L., Dark Currents in Long Wavelength Infrared HgCdTe Gated Photodiodes. Journal of Electronic Materials. 33(6): p. 621. (2004)

[19] Gilmore, A.S., Bangs, J., and Gerrish, A., Current voltage modeling of current limiting mechanisms in $\mathrm{HgCdTe}$ focal plane array photodetectors. Journal of Electronic Materials. 34(6): p. 913-921. (2005)

[20] Antoszewski, J., Musca, C.A., Dell, J.M., and Faraone, L., Small two-dimensional arrays of midwavelength infrared $\mathrm{HgCdTe}$ diodes fabricated by reactive ion etching-induced $p$-to-n-type conversion. Journal of Electronic Materials. 32(7): p. 627-632. (2003)

[21] Kinch, M.A., Chandra, D., Schaake, H.F., Shih, H.-D., and Aqariden, F., Arsenic-Doped MidWavelength Infrared HgCdTe Photodiodes. Journal of Electronic Materials. 33(6): p. 590. (2004)

[22] Haakenaasen, R., Steen, H., Lorentzen, T., Trosdahl-Iversen, L., Van Rheenen, A.D., and Syversen, H., Planar n-on-p ion milled mid-wavelength and long-wavelength infrared diodes on molecular beam epitaxy vacancy-doped CdHgTe on CdZnTe. Journal of Electronic Materials. 31(7): p. 710-714. (2002)

[23] Shaw, D. and Capper, P., Conductivity type conversion in $\mathrm{Hg}_{1-x} \mathrm{Cd} d_{x} \mathrm{Te}$. Journal of Materials Science-Materials in Electronics. 11(2): p. 169-177. (2000) 


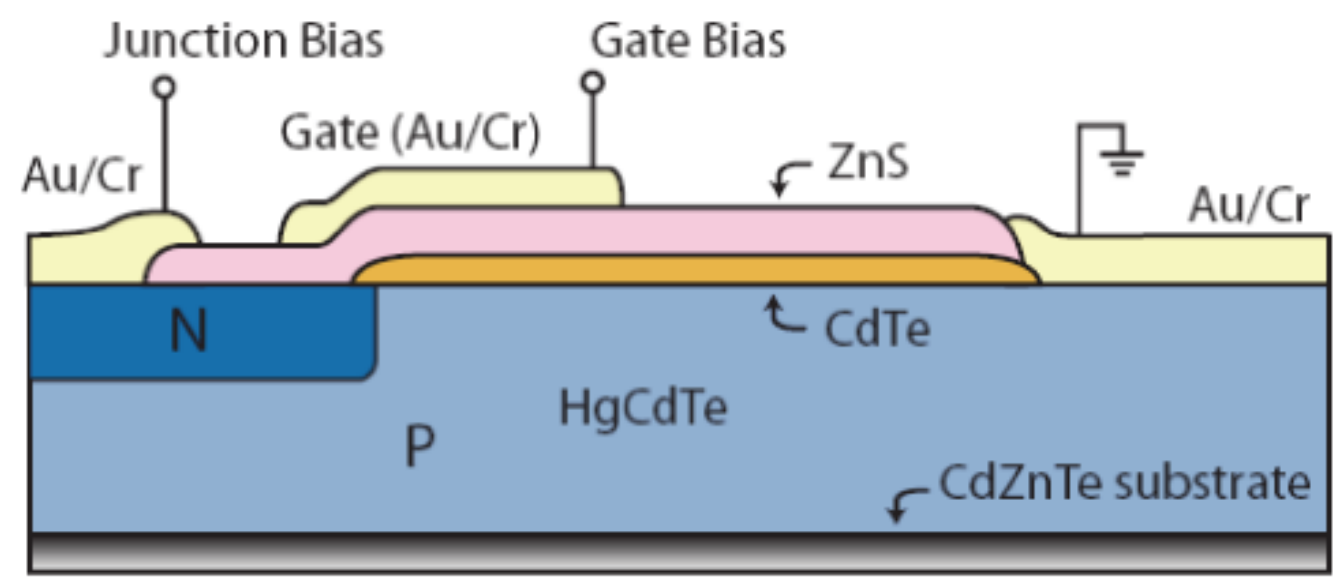

Fig. 1 - Cross section of gated photodiode structure. Circular junction diameter of $300 \mu \mathrm{m}$ or $410 \mu \mathrm{m}$.

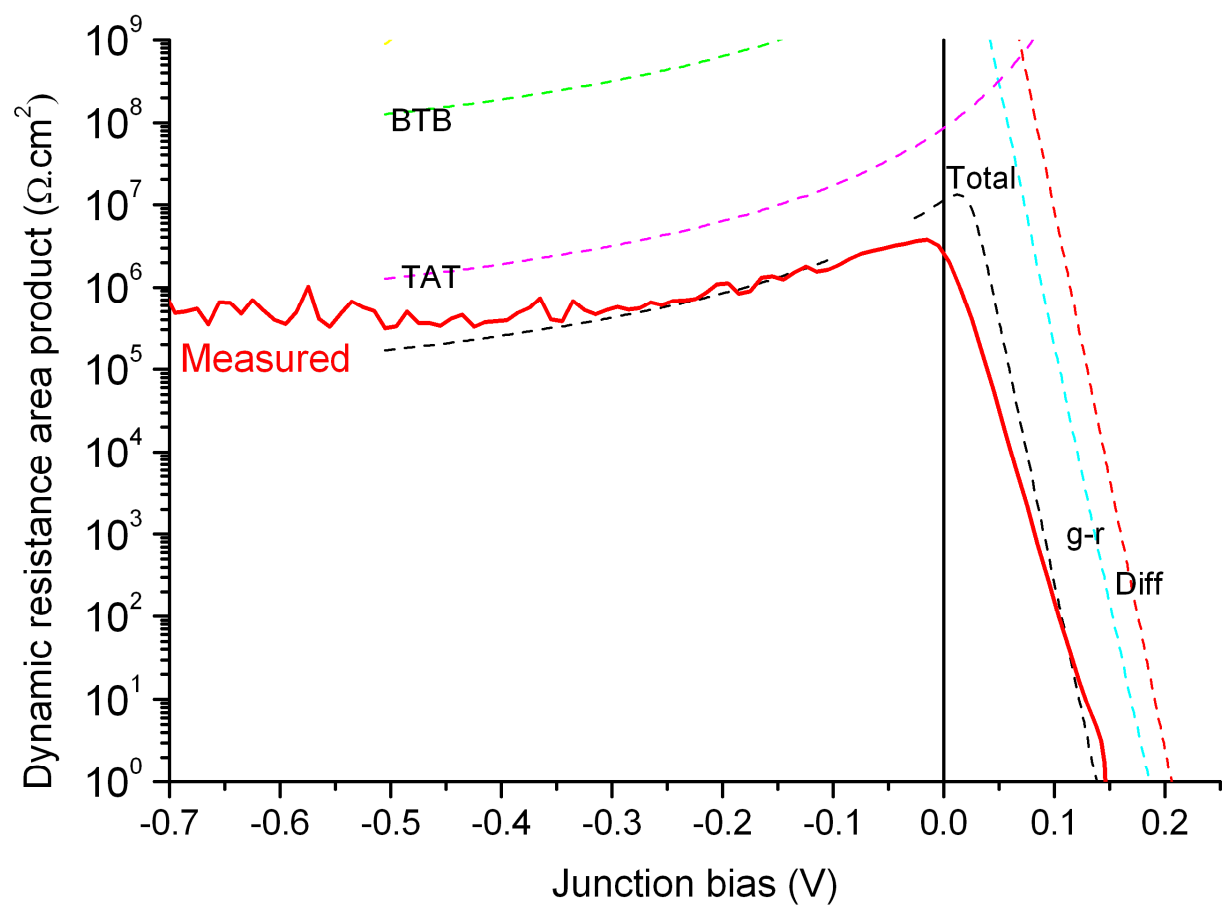

Fig. $2-R_{d} A$ for photodiode $A 01$ (diameter $410 \mu \mathrm{m}$ ) from array $\mathrm{C}$ measured at $77 \mathrm{~K}$ in the dark. Dotted lines show the modeled behaviour of dark current mechanisms. 


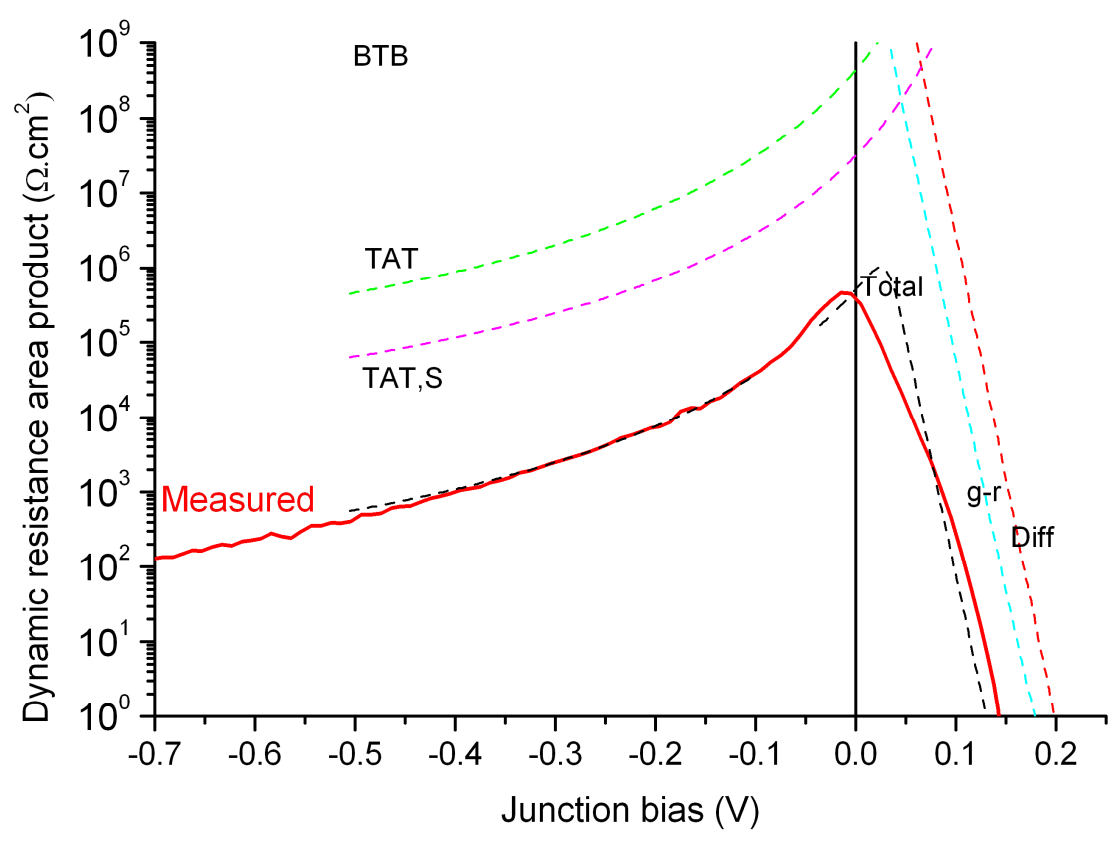

Fig. $3-R_{d} A$ for diode A02 (diameter $410 \mu \mathrm{m}$ ) from sample B measured at $77 \mathrm{~K}$ in the dark. Dotted lines show the modeled behaviour of dark current mechanisms.

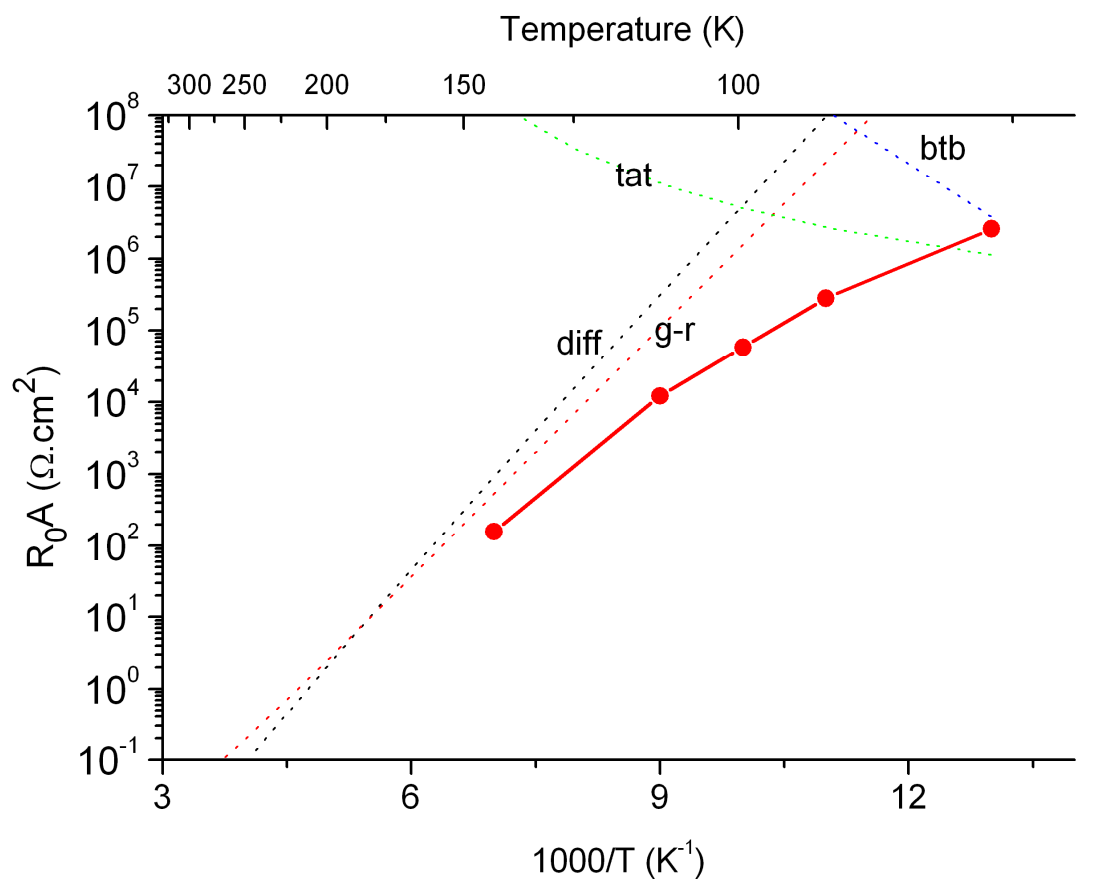

Fig. 4 - Temperature dependence of $R_{0} A$ for photodiode $A 01$ from sample C. Dotted lines show the modeled behaviour of the dark current mechanisms. 


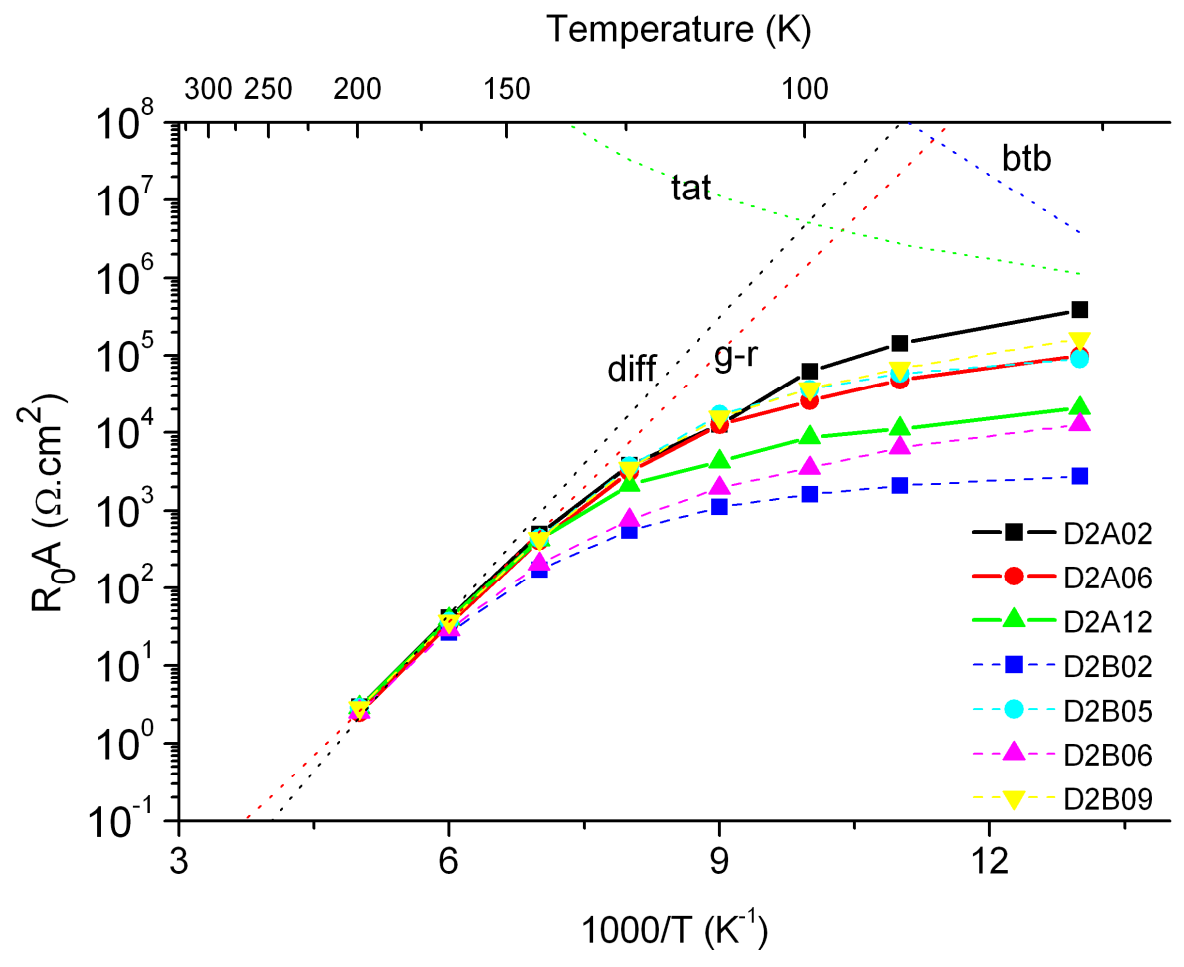

Fig. 5 - Temperature dependence of $R_{0} A$ for various photodiodes from sample array $B$.

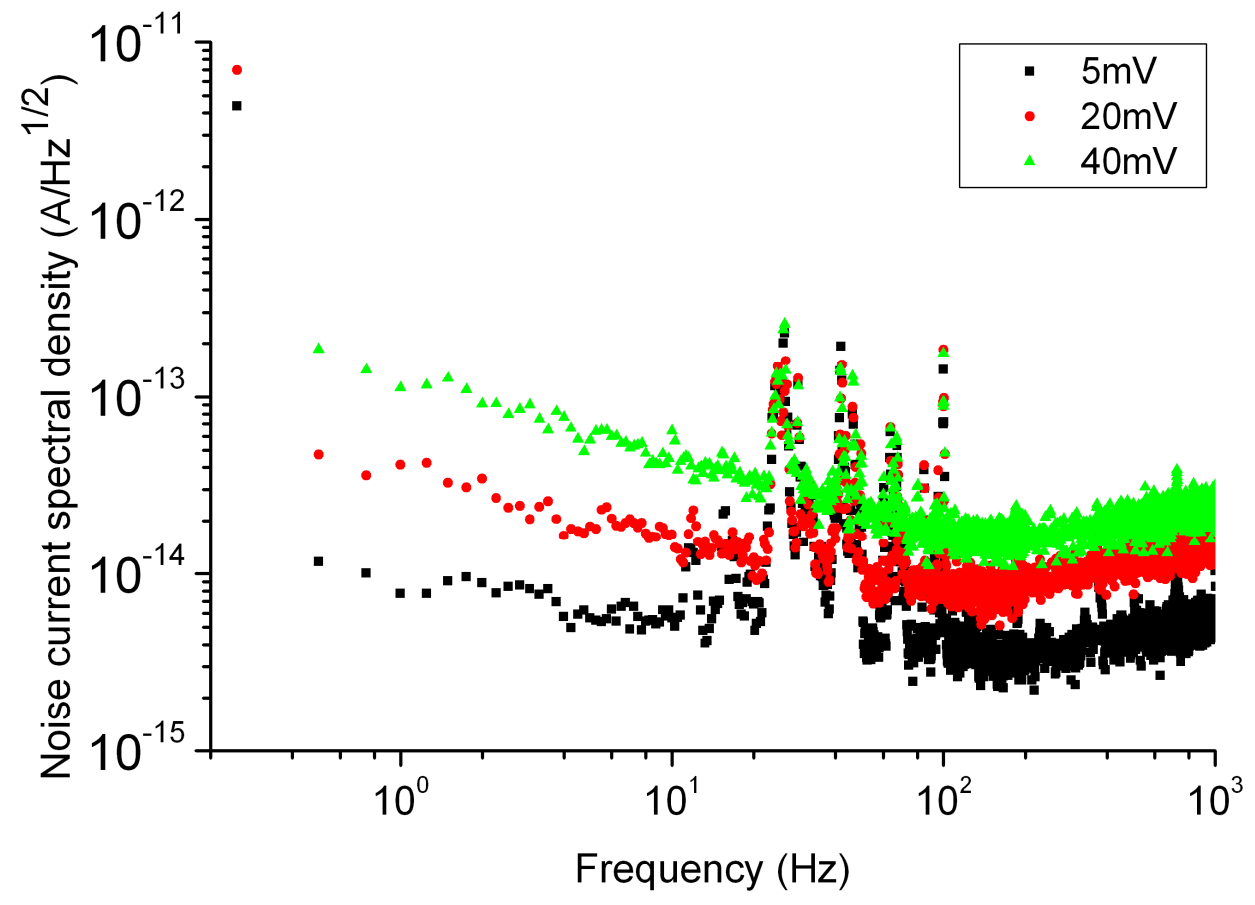

Fig. 6 - Noise characteristics of photodiode A01 from sample C measured at 77K in the dark. 


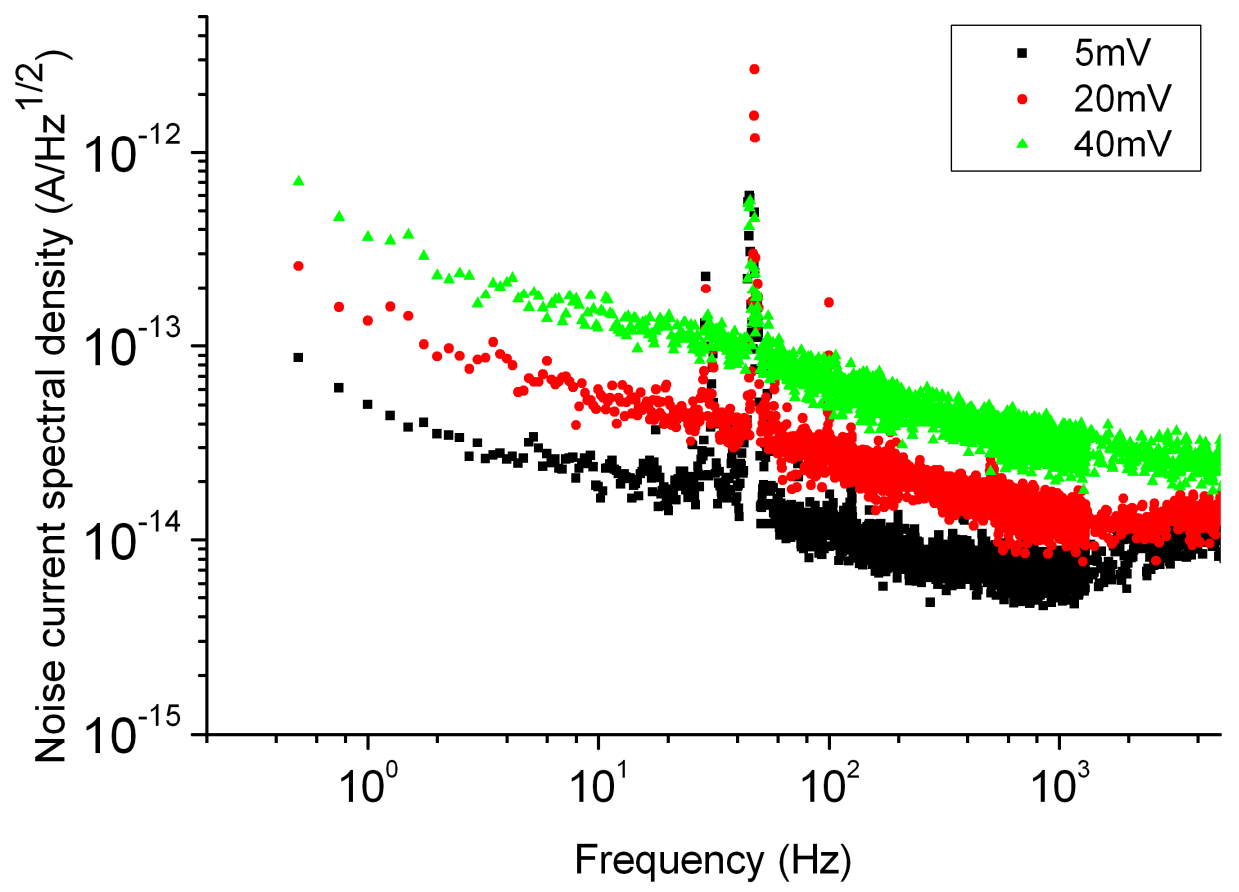

Fig. 7 - Noise characteristics of photodiode A02 from sample B measured at 77K in the dark. 